\title{
Smalti Murano Glass Tessella's Applied Outdoor in the Trencadís Catalan Modernism Mosaic: Recognition of Preliminary Alteration Patterns
}

\author{
Raül Serra i Fabregà ${ }^{1}$, Joan Ll. Zamora i Mestre ${ }^{2}$ and Paloma Arias ${ }^{3}$
}

${ }^{1} \mathrm{PhD}$ in Architecture, Building and Urban Technology, Universitat Politècnica de Catalunya (UPC) - BarcelonaTECH, ETSAB, Av. Diagonal, 649, 08028-Barcelona, Spain, raul.serra@upc.edu

${ }^{2}$ Laboratori d'Innovació i Tecnologia de l'Arquitectura (LiTA), Universitat Politècnica de Catalunya (UPC) - BarcelonaTECH, Pere Serra, 1-15, 08173-Sant Cugat del Vallès, Spain, joan.1luis.zamora@upc.edu

${ }^{3}$ Programa de Doctorado Tecnología de la Arquitectura, de la Edificación y del Urbanismo Universitat Politècnica de Catalunya (UPC) - BarcelonaTECH, Av. Diagonal, 649, 08028- Barcelona, Spain,paloma.arias@upc.edu

\begin{abstract}
This contribution presents previous results of a study carried out with the aim of achieving better results in the restoration processes by replacing the tiles of an exterior mosaic tiling of the Trencadis type of Catalan Modernism. In this case the trencadis tiles come from the cutting of pieces, called piastras, of opaque and mass-colored glass called Smalti Murano glass. These companies have extensive color catalogs that, although not exactly the same as they were a century ago, are close enough for these replacement tasks. Each color corresponds, however, to a different composition of glass and a slightly different manufacturing process. These differences favor that the differences of color always go accompanied also of differences of superficial aspect. However, not all color references currently available have the same surface quality since this product, during its manufacture, acquires primary defects typical of artisanal processes. So far the predominant criteria for the selection of the new references of piastras that must replace the product damaged by the extensive exposure to the weather as a coating are based primarily on color. However, durability requirements must also be part of the selection criteria for substitution references. The primary defects that a product already presents in its superficial quality are often indicators of either a more irregular quality or of circumstances that favor the early appearance of secondary defects throughout its useful life. The main objective of this study is to ensure, in restoration work by substitution, the selection of the best piastras from the point of view of surface quality, to apply on site the most similar tiles of tone and with greater durability. This study is part of a collaboration between the study group LiTA (Laboratori d'Innovació i Tecnologia de l'Arquitectura) (UPC) and a private non-profit organization.
\end{abstract}

Keywords: Colour, Murano Glass, Smalti, Trencadís, Preliminary Alteration Patterns. 


\section{Introduction}

Murano Smalti glass has been used for centuries as a tile in colored mosaics as a coating for interior walls. Smalti glass, by its nature is very bright, chemically stable and has a solid color mass. Given its intense mass coloration, the glass tiles were placed sideways with what they always had small dimensions (of the order of the thickness of the glass) and parallel faces.

The trencadís is a coating typical of the architecture of Catalan Modernism that is composed of small pieces of materials, irregularly cut, such as enameled ceramics or marble, arranged randomly supported by its main face (table) on a layer of mortar forming a colorful polygonal mesh that has three-dimensional architectural motifs. It thus differs from the traditional historical mosaic by the arbitrary arrangement of the carved tiles with irregular shapes, by its ability to solve the coating of three-dimensional surfaces and because it is very often in situations of full weather where it manifests itself with all its brightness and color. (Fig. 1)

The uniqueness of Murano Smalti glass is due to the fact that this mass-colored opal glass is still handcrafted today using traditional techniques. The same techniques used during Catalan Modernism. The producing companies continue to supply hundreds of different colors that cover almost all shades.
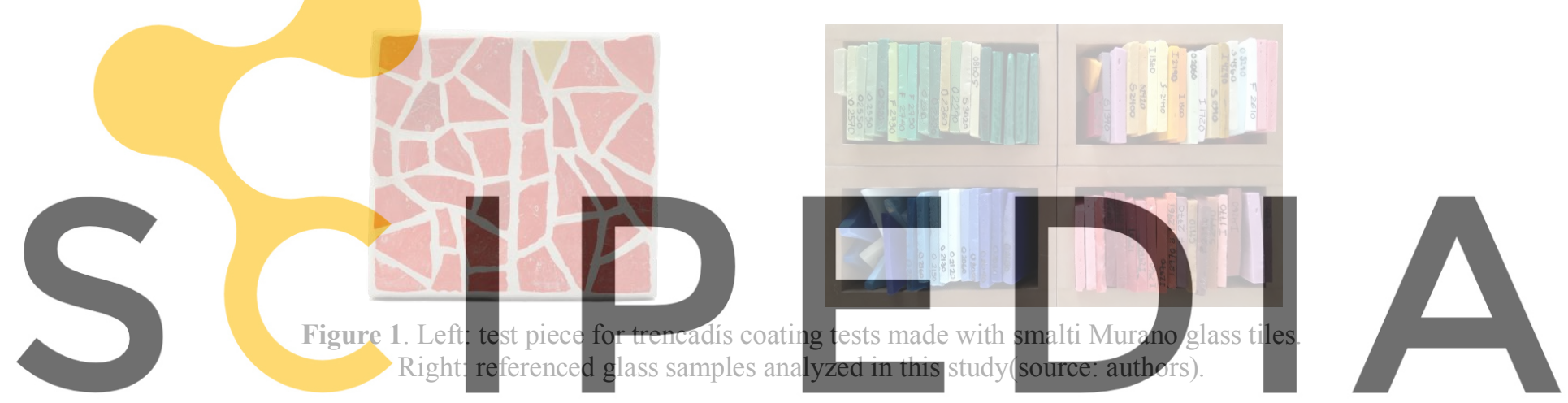

\section{The Material}

Register for free at https//www.scipedia.com to download the version without the watermark In this study the piastras are made of opal glass, called Smalti. In the world of glass mosaics, smalti indicates a bright, opaque, mass-colored glass with high lead content, generally obtained by adding a crystalline material (corpo) and colored crystals (anima) to a molten transparent glass.
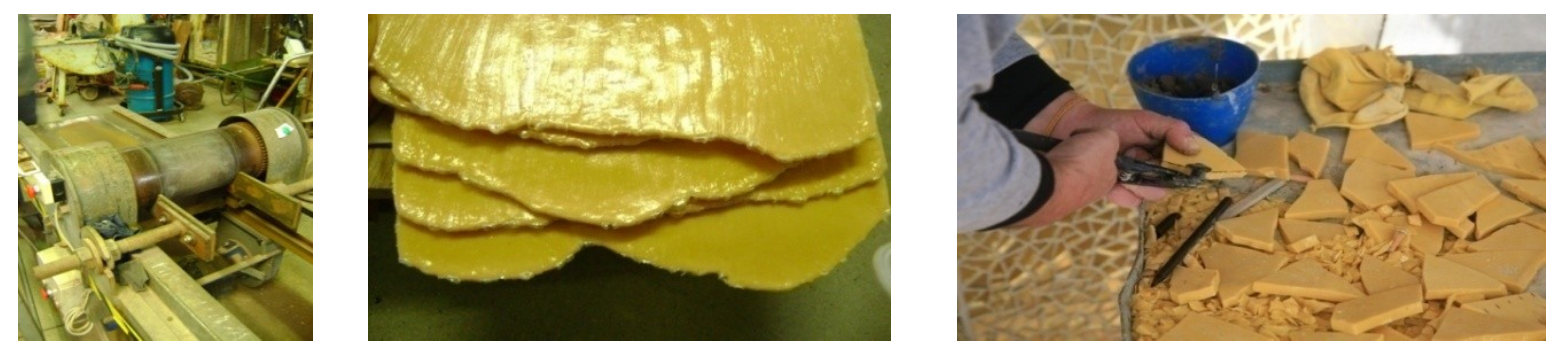

Figure 2. Left: Murano Smalti glass forming process by lamination until flat piastras are obtained. Center: glass piastras shown on Side 1. Right: manual cutting process (source: authors). 
The traditional coloring agents commonly used for glass are a limited number of metal ions which, by absorbing part of the incident light, give rise to a great diversity of colorations. The apparent color of each glass depends on the nature of the ion, its oxidation state and the composition of the glass in which it melts.

Once the desired mixture is mixed in the corresponding crucible, homogenized and reached the appropriate temperature, masses of molten material are removed from the oven to form the piastras.

During the process of mixing, melting, forming and subsequent cooling of the piastras, they acquire various primary defects that alter their surface quality. These primary defects not only devalue their appearance but can also reduce their durability expectations as they are the source of new secondary anomalies that appear with the passage of time and exposure to the weather.

Due to the glass forming process, the surface quality obtained on both sides is not the same as that the face in contact with the table is kept in contact with the metal while the face in contact with the laminator is kept in contact with the atmosphere of the premises.

Since the face of the Murano glass in the trencadís lining will not be the edge but the table of the piastra, it will be necessary to select the one with the least primary defects present. This is how the face of each piastra is seen, called (A) in this study and the hidden face of each piastra, called (B) in this study.

The visual difference (brightness, color, surface homogeneity) of the faces is significant and should be taken into consideration at the time of restoration, since it can alter the assessment of the final overall quality of the trencadís lining.
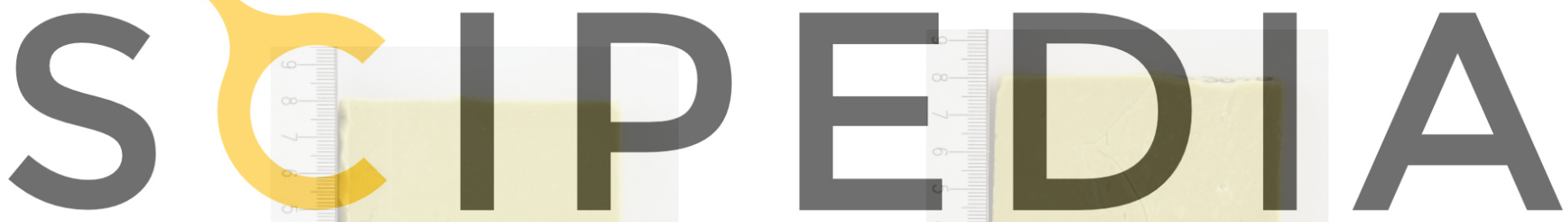

Register for free at https//www.scipedia.com to download the version without the watermark
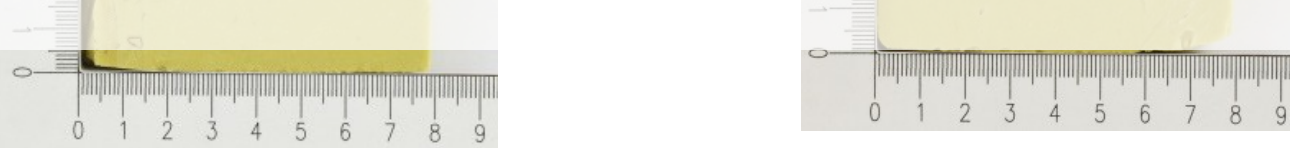

Figure 3. On the left the Face (A) of the reference glass S 3020 and on the right the Face (B) of the same glass reference where bubbles and folds often present in the hidden face of these glasses are appreciated (source: authors).

The objective of this research is to develop a procedure that allows grouping and ordering the various references available according to their apparent surface quality, so that artisans can select and use those references that, for the same color, have fewer primary defects. In order to favor this objective, the following inspection methodology, census and assessment of surface quality were proposed. 


\section{Methodology}

With this objective a campaign was made to capture digital images on a universe of 77 references and 2 pieces for each reference, of approximate dimensions $8 \times 8 \mathrm{~cm}$, which constitute the basic sample ceded for this study by a company mainly oriented to the elaboration of this product.

Each of the 77 references has a different tonality, but these can be grouped into 11 color ranges:

Brown (17 references), violet (3 references), white (5 references), blue (11 references), green (16 references), orange ( 7 references), yellow (5 references), red (5 references), pink (4 references), black (2 references) and gray (2 references).

The samples are identified according to the manufacturer's catalog code. These codes are composed of an initial letter and 4 numbers, the letters refer to the type of smalti, differentiating them into: C: Skin (3 references), F: Smalti Fino (9 references), I: Imperial (25 references), OR: Ordinary Smalti (28 references), S: Special (12 references), and the numbers below identify the hue of the color

This leads us to show that the same color range can be found in different types of smalti glass. Since the same color can be prescribed in different types of glass, it is about adopting the one that presents less primary defects and that is probably why it will age more adequately throughout its useful life, increasing its durability.

\subsection{Digitization}

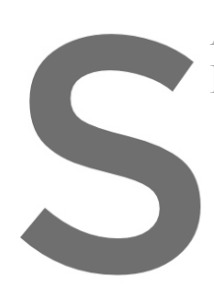

All received parts $h$

F2200 series" model. T

- Cleaning the face

removal of the identi

- Placement of the p

20 pieces chosen at $r$
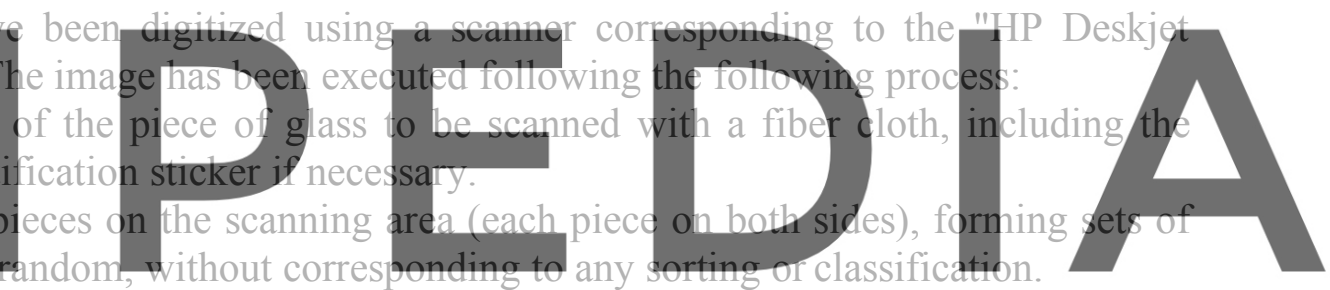

- Scan of the set of parts, using the software "HP Solution Center", typical of the hardware

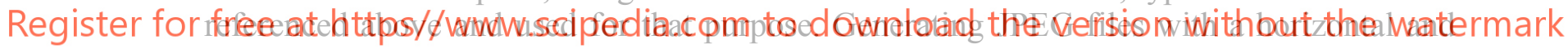
vertical resolution of $600 \mathrm{dpi}$ and a variable dimension, about $10 \mathrm{MB}$.

\subsection{Digital Image Editing}

The JPG files corresponding to the scans of the groups of 20 glass samples and the subsequent edition of the images of the individual samples have been treated with the software "Adobe Photoshop CS6 version: 13.0.1 service pack: 1".The result of this work was the images of individual pieces, making a distinction between the two faces of each piece and calling them "sight" for the face generated by having a work view (face A), and "hidden" for the face generated to be arranged in contact with the mortar (face B). Generating jpg files of $1146 \mathrm{x}$ 1146 pixels and a horizontal and vertical resolution of $600 \mathrm{dpi}$. 

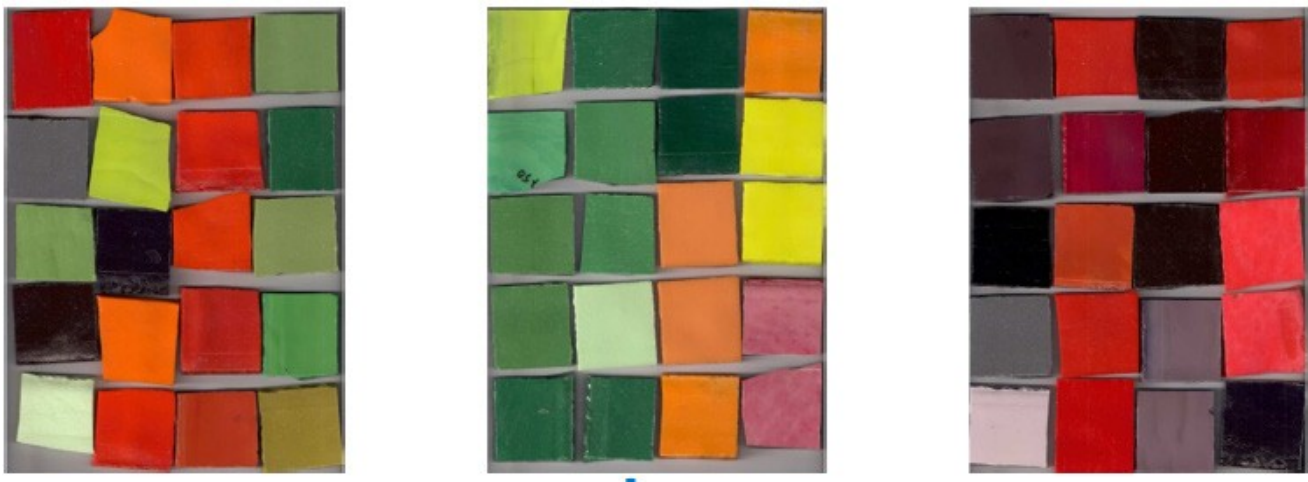

Figure 4.Overall images of the various image scanning processes with flatbed scanner (source: authors).

\subsection{Characterization of the Identifying Color of the Samples}

The characterization of the identifying color of the samples has been made from the images obtained in the previous process, and responds to the following sequence:

- superposition of 5 arbitrary markers on all images of individual samples.

- measurement of RGB color values in the center of each marker superimposed on the

image of the sample piece, with the aim of parameterizing its color.

- overlay the histogram, in each of the images of individual samples.
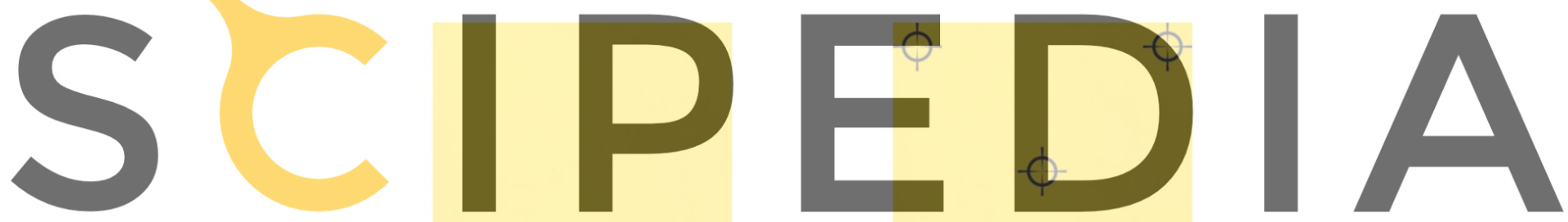

Register for free at https//www.scipedia.com to downłoad the versiobwithout the watermark

\begin{tabular}{|c|c|c|c|}
\hline POINT 1 & 250 & 218 & 20 \\
\hline POINT 2 & 246 & 217 & 27 \\
\hline POINT 3 & 248 & 218 & 21 \\
\hline POINT 4 & 244 & 216 & 18 \\
\hline POINT 5 & 244 & 214 & 29 \\
\hline $\begin{array}{l}\text { AVERAGE } \\
\text { VALUATION }\end{array}$ & 246 & 217 & 23 \\
\hline
\end{tabular}

Step 3: Measure the RGB color values in the center of each marker
Step 2: Overlay of 5 markers

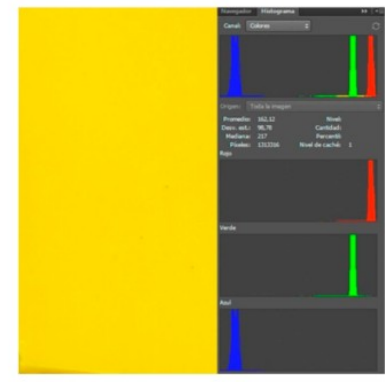

Step 4: Overlay the histogram

Figure 5. Model type of file for the evaluation of the color of the sample (source: authors). 


\subsection{Sample Defect Assessment}

To assess the visible surface quality of each sample on each face:

- An initial value of 10 has been granted to each face

- For each face of each piece examined, a qualitative assessment of each defect has been made, giving a value of -1 if the said defect is generalized or 0 if it is punctual or nonexistent.

\subsection{Census of Surface Defects}

The 10 primary defects observed on this face during the visual inspection are listed below, accompanied by their description:

- A1 Scales: Presence of half uncoiled sheets of the sample piece.

- A2 Fissures: Presence of marked fissures in the observed face of the sample.

- A3 Cracks: Presence of lesions with differently separated lips and variable depth.

- A4 Polygonal microfisuration: Presence of microcracks showing a cracking structure on

the observed face.

- A5 Wound: Presence of discontinuities more or less deep and with well-defined lateral

limits.

- A6 Longitudinal mark: Presence of unidirectional longitudinal marks, often accompanied

by air pockets and very elongated spots.

- A7 Stain: Presence of spots of different coloration, more or less rounded.
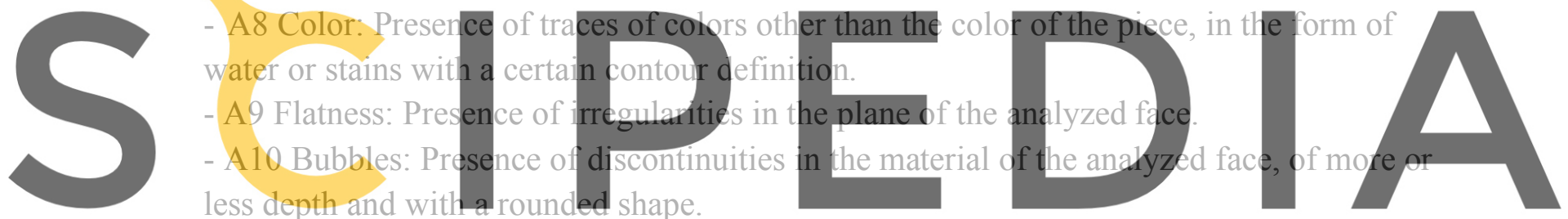

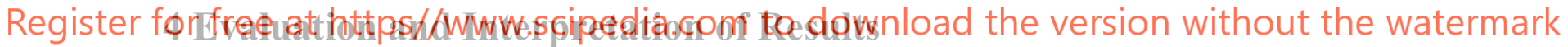

The first interpretation of the results (Table 1) shows that there are different evaluations:

Table 1. Average concurrence of defects on both faces.

\begin{tabular}{lcc}
\hline & View face (A) analysis & Hidden face (B) analysis \\
\hline Concurrence of more than 5 defects & $6.17 \%$ & $24.69 \%$ \\
\hline Concurrence of 5 defects & $20.99 \%$ & $22.22 \%$ \\
\hline Concurrence of 4 defects & $22.84 \%$ & $17.90 \%$ \\
\hline Concurrence of 3 defects & $21.60 \%$ & $24.07 \%$ \\
\hline Concurrence of 2 defects & $18.52 \%$ & $7.41 \%$ \\
\hline Presence of a single defect & $7.41 \%$ & $3.70 \%$ \\
\hline Without any defect & $2.47 \%$ & $0 \%$
\end{tabular}

About the analysis between different samples of a same reference, the average additional defects on both faces (Table 2) are the following: 
Table 2. Average additional defects on both faces.

\begin{tabular}{lcc}
\hline & View face $(\mathrm{A})$ analysis & Hidden face (B) analysis \\
\hline The same defects are found & $30.86 \%$ & $33.33 \%$ \\
\hline There is 1 additional defect & $35.80 \%$ & $32.10 \%$ \\
\hline There are 2 additional defects & $12.35 \%$ & $14.81 \%$ \\
\hline There are 3 additional defects & $14.81 \%$ & $13.58 \%$ \\
\hline There are 4 additional defects & $6.17 \%$ & $2.47 \%$
\end{tabular}

Since the objective of the study was to order the different references observed according to their surface quality (primary defects), to offer information to the artisan before the task of transferring the color's design choice to the product code for the highest quality execution, has been considered a new variable that corrects the scores regarding the characterization of this material. This variable is the variability of defects observed in different samples of the same code.

Thus, understanding that a lower variability of valuations between different samples of the same code is preferable, from the point of view of the reliability of the valuation performed, and a greater variability of valuations is inconvenient due to the lower reliability of the same valuation, the criterion of correcting the results has been adopted according to the following criteria:

Table 3. Criteria of modification of the sample's score.

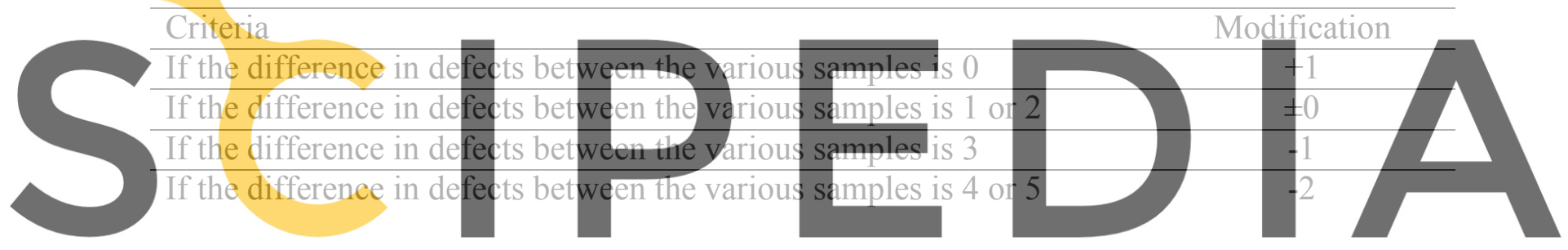

\section{Final Qualification of References}

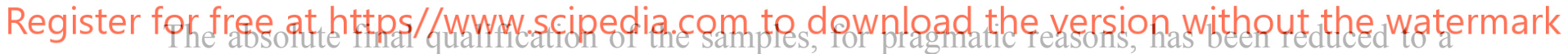

double score for each company code: a preponderant score for the face and an accessory score

for the hidden face, based on a 5-star system such as maximum and 1 star minimum. To capture this fact graphically, generic files have been formulated that contain the information for each code of the company analyzed.

Finally, a relative final qualification of the samples with respect to the results of the samples belonging to codes of the same color range has been established. The objective is to help the selection of codes in the translation of the design color to the nomenclature of the execution material:

- A high quality code, that is, the best quality available (green color rating),

- A medium quality code (orange rating): its substitution is recommended with the recommended green references ordered.

- A low quality code (red rating): mandatory replacement is prescribed by the ordered orange references. 
About the references of Murano glass, the results are as follows:

Table 4. Average distribution of quality by smalti types.

\begin{tabular}{l|c|c|c|c|c}
\hline & C: Skin & F: Fine & I: Imperial & O: Ordinary & S: Special \\
\hline High quality & $0 \%$ & $0 \%$ & $20 \%$ & $14.29 \%$ & $8.33 \%$ \\
\hline Medium quality & $100 \%$ & $88.89 \%$ & $60 \%$ & $53.57 \%$ & $50 \%$ \\
\hline Low quality & $0 \%$ & $11.11 \%$ & $20 \%$ & $32.14 \%$ & $41.67 \%$
\end{tabular}

And in terms of color spectrum:

Table 5. Average distribution of quality by colours.

\begin{tabular}{l|c|c|c|c|c|c|c|c|c|c|c}
\hline Quality & Brown & Violet & White & Blue & Green & Orange & Yellow & Red & Pink & Black & Grey \\
\hline High & $11.76 \%$ & $33.33 \%$ & $40 \%$ & $9.09 \%$ & $0 \%$ & $14.29 \%$ & $60 \%$ & $0 \%$ & $0 \%$ & $0 \%$ & $0 \%$ \\
\hline Medium & $52.94 \%$ & $66.67 \%$ & $40 \%$ & $54.55 \%$ & $68.75 \%$ & $57.14 \%$ & $40 \%$ & $60 \%$ & $100 \%$ & $100 \%$ & $100 \%$ \\
\hline Low & $35.28 \%$ & $0 \%$ & $20 \%$ & $36.36 \%$ & $31.25 \%$ & $28.57 \%$ & $0 \%$ & $40 \%$ & $0 \%$ & $0 \%$ & $0 \%$
\end{tabular}

\section{Conclusions}

Murano Smalti glass has been revealed as a material with a high amount of concurrent surface primary defects, defects that affect similarly to its two faces, although they predominate on its face (B). It can be concluded because most of the primary defects observed are inherent to the product as it is currently produced and few others are inherent to the face (B), which is in contact with the forming table.

The results of this preliminary study, although they illustrate quite clearly the primary defects that this material suffers, should still be considered indicative, since several limiting causes must be considered:

- these are parts fron selected by the manu - these are cut pieces of larger piastras; In

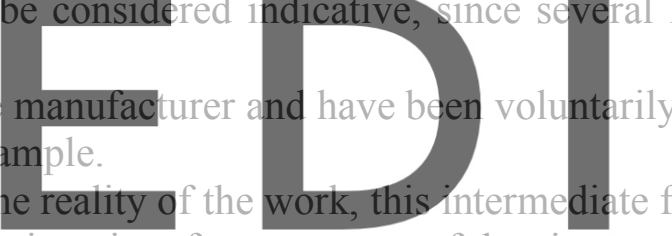

is not used, but each tile is extracted, by fractionation, from any area of the piastra.

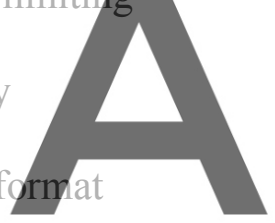

Register for free at het of references and golors analyzed in each range is not.equivalent. ORCIID

Raül Serra Fabregà: http://orcid.org/0000-0002-5500-1858

Joan Ll. Zamora: http://orcid.org/0000-0002-7705-6171

Paloma Arias: http://orcid.org/0000-0002-8334-8269

\section{References}

Arias, P. and Zamora, J. (2018) Evaluación de las características cromáticas del vidrio de Murano utilizado en revestimientos exteriores de trencadis.Euro-American Congress on Construction Pathology, Rehabilitation Technology and Heritage Management,7th REHABEND Congress: Caceres (Spain), May 15th-18th. Santander (Cantàbria): Universidad de Cantabria, 2018, p. 1254-1263. http://hdl.handle.net/2117/131486

Gómez, J. (2015) Los pináculos de la Sagrada Familia: restauración y obra nueva (Tesis doctoral) Universitat Politècnica de Catalunya. http://hdl.handle.net/2117/95689

Verità, M., James, L., Freestone, I., Henderson, J., Nenna, M.-D. and Schibille, N. (2009), Glossary of Mosaic Glass Terms, edited by B. Bjornholt (Centre for Byzantine Cultural History, University of Sussex, 2009)

Verità, M. (2000) Technology and deterioration of vitreous mosaic tesserae, Studies in Conservation, 45: sup 3, 65-76, DOI: $10.1179 /$ sic.2000.45.s3.007. 